\title{
1 Abundance and community composition of comammox bacteria in different 2 ecosystems by a universal primer set
}

3

4 Zhirong Zhao ${ }^{\mathrm{a}}$, Guohe Huang ${ }^{\mathrm{a}}$, Mingyuan Wang ${ }^{\mathrm{a}}$, Nan Zhou ${ }^{\mathrm{a}}$, Shishi He ${ }^{\mathrm{a}}$, Chenyuan Dang ${ }^{\mathrm{b}}$,

$5 \quad$ Jiawen Wang ${ }^{\mathrm{b}}$, Maosheng Zheng ${ }^{\mathrm{a}}{ }{ }$

6

$7 \quad{ }^{a}$ MOE Key Laboratory of Resources and Environmental System Optimization, College of

8 Environmental Science and Engineering, North China Electric Power University, Beijing

9 102206, China

$10{ }^{b}$ The Key Laboratory of Water and Sediment Sciences, Ministry of Education, Department of

11 Environmental Engineering, Peking University, Beijing 100871, China

21 Corresponding author:

22 Maosheng Zheng;

23 E-mail: maoshengzheng@ncepu.edu.cn

24 Address: Main Building G915, North China Electric Power University, Beijing 102206, China 


\section{Abstract:}

26 Complete ammonia oxidizing bacteria (CAOB) have been recognized as a new member of ammonia-oxidizing microorganisms (AOMs) due to its single-step nitrification capability. However, the abundance and diversity of CAOB in environmental ecosystems were still far from known owing to the lack of specific molecular marker. Herein, a universal primer set specifically targeting the both clades of $\mathrm{CAOB}$ amoA gene with high specificity and coverage was successfully designed. Intriguingly, real-time quantitative PCR tests revealed that CAOB were ubiquitous and unexpectedly abundant in agricultural soils, river sediments, intertidal zones, drinking water and wastewater treatment systems. Phylogenetic analysis indicated that clade A existed in all the five types of ecosystems, while clade B were only detected in soil and sediment samples. Four sub-clusters were further classified within clade $\mathrm{A}$, in which $N$. nitrosa cluster dominated CAOB amoA in activated sludge samples while the new recognized soil cluster was the primary constitute in soils. Moreover, the niche specialization between different $\mathrm{CAOB}$ species and the environmental conditions were supposed to be the primary driven force to shape the diversity and community of CAOB. This study provided a strong evidence in support of the ubiquities and high abundances of $\mathrm{CAOB}$ in various environmental ecosystems and highlighted the significance of including CAOB as the new member of AOMs to re-evaluate the biogeochemical nitrogen cycle.

Keywords: Comammox; Primer; Abundance; Community; ammonia-oxidizing 


\section{Introduction}

Nitrogen is an essential element for nucleic acids and proteins synthesis in all living organisms and the microorganism-mediated nitrogen cycle plays a critical role in the existence and sustainability of life. Ammonia oxidation to nitrite catalyzed by ammonia-oxidizing archaea ( $\mathrm{AOA})$ and bacteria ( $\mathrm{AOB})$ was recognized as the first and rate-limiting step of nitrification, followed by nitrite oxidation to nitrate by nitrite-oxidizing bacteria (NOB). This division of labor of ammonia oxidizing microorganisms (AOMs) and NOB has been long-held accepted as the only nitrification theory in the biogeochemical nitrogen cycle for more than 120 years since the discovery of AOB. Nevertheless, it was theoretically feasible to oxidize ammonia to nitrate by one single microbe, as complete ammonia oxidation (comammox) was more energy efficient than the traditional two-step nitrification process (Costa et al., 2006). This theory had not been experimentally testified until three complete ammonia-oxidizing bacteria (CAOB) capable of independently performing complete nitrification were enriched (Daims et al., 2015; Kessel et al., 2015).

Intriguingly, based on $16 \mathrm{~S}$ rRNA gene all the three $\mathrm{CAOB}$ strains were phylogenetically affiliated with genus Nitrospira, which was the most abundant and widespread genus of NOB (Daims et al., 2016; Koch et al., 2015). However, CAOB were confirmed to possess a whole set of nitrifying genes encoding for ammonia monooxygenase (AMO), hydroxylamine dehydrogenase (HAO) and nitrite oxidoreductase (NXR), providing strong evidences for the capability of performing complete ammonia oxidation to nitrate via nitrite (Daims et al. 2015; van Kessel et al. 2015; Camejo et al. 2017). CAOB amoA gene formed a distinct phylogenetic cluster with a low similarity to the canonical $\mathrm{AOB}$ amoA, suggesting it was an ideal phylogenetic biomarker to identify and quantify $\mathrm{CAOB}$ in complex microbial communities. In addition, $\mathrm{CAOB}$ amoA gene was divergent which could be divided into two monophyletic clades, clade A and clade B (Pjevac et al., 2017).

To date, widespread attentions had been attracted on comammox owing to their potential contribution in ammonia oxidation and significant role in nitrogen cycle. The wide distribution of $\mathrm{CAOB}$ have been suggested through metagenomic surveys in some environmental habitats, such as paddy fields, agricultural soils, freshwater habitats and wastewater treatment systems (Kessel et al., 2015). High proportions of $\mathrm{CAOB}$ amoA gene were found within all the copper-containing membrane-bound monooxygenase (CuMMO) in nine environmental samples through a high degenerate primer (Wang et al., 2017a). Due to the divergence of the new amoA gene, two sister PCR primer sets targeting respective clade A and B were designed 
and used to explore the abundance and diversity of CAOB in different ecosystems (Pjevac et al., 2017). A newly designed primer set specifically targeting the clade A of CAOB amoA gene revealed high abundances of CAOB in full-scale WWTPs (Wang et al., 2018). Likewise, a clade A targeted primer set was designed to quantify the $\mathrm{CAOB}$ abundances in various environments (Xia et al., 2018).

However, the numerical abundances and community composition of CAOB in different ecosystems were still far from known. To fill this gap, the universal PCR primer set targeting both clade A and clade $\mathrm{B}$ of $\mathrm{CAOB}$ amoA was successfully designed in this study. With the specific amplification, the abundances and diversity of CAOB in five types of environmental habitats were investigated through quantitative PCR and high-throughput sequencing technology, respectively. This study provided an efficient molecular tool, which would facilitate the follow-up researches on comammox.

\section{Materials and methods}

\subsection{Primer design and evaluation}

PCR primer pairs targeting both clade $\mathrm{A}$ and clade $\mathrm{B}$ of $\mathrm{CAOB}$ amoA gene were designed to detect the presence of $\mathrm{CAOB}$ in diverse environmental samples. Briefly, putative $\mathrm{CAOB}$ amoA sequences affiliated with clade A or clade B were downloaded from National Center for Biotechnology Information (NCBI) as the query sequences. After clustering at 0.99 similarity level, a database including 120 putative $\mathrm{CAOB}$ amoA sequences were used to design the primer set.

To evaluate the specificity of the newly designed primer, the amoA database containing 43876 sequences and pmoA database containing 44402 sequences were downloaded from FunGene database. Then the phylogenetic tree was constructed using R, MAFFT, Xshell and FigTree software. The sequences that did not classified into the two genes were deleted to construct the accurate amoA and pmoA database containing 30963 and 31044 sequences, respectively. Primer coverage under $0,1,2$ mismatch were calculated using the program primersearch of EMBOSS v6.5.7.0.

\subsection{Collection of environmental samples}

Eighteen samples were collected from five types of environmental ecosystems including agricultural soil, river sediment, drinking water, intertidal zone and activated sludge samples. All samples were immediately transported to the laboratory in ice and stored at -20 $\square$. Detailed descriptions and detailed parameters of these samples were listed in Table 1. 


\subsection{DNA extraction and quantitative PCR}

Total genomic DNA was extracted from each sample using the FastDNA Spin Kit for Soil (MP Biomedicals, USA) according to the manufacturer's instructions.

The abundances of $\mathrm{AOA}, \mathrm{AOB}$ and $\mathrm{CAOB}$ amoA genes were quantified with archamoA 19f/616r (Pester et al., 2012), amoA 1F/2R (Rotthauwe et al., 1997) and the present designed comamoA F/R on Applied Biosystems 7500 system, respectively. Quantitative PCR were performed in a $20 \mu \mathrm{L}$ system consisted of $10 \mu \mathrm{L} 2 \times$ SuperReal Premix Plus, $2 \mu \mathrm{L}$ 50×Roxpreference Dye, $0.5 \mu \mathrm{L}$ forward and reverse primers, $2 \mu \mathrm{L}$ template DNA and $5 \mu \mathrm{L}$ RNase-free $\mathrm{H}_{2} \mathrm{O}$.

The AOA and CAOB amoA gene were amplified under the same condition: an initial denaturation step at $95{ }^{\circ} \mathrm{C}$ for $15 \mathrm{~min}$, followed by 40 cycles of denaturation at $95{ }^{\circ} \mathrm{C}$ for $30 \mathrm{~s}$, annealing at $53{ }^{\circ} \mathrm{C}$ for $30 \mathrm{~s}$ and extension at $72{ }^{\circ} \mathrm{C}$ for $1 \mathrm{~min}$. For AOB amoA gene and $16 \mathrm{~S}$ rRNA, the same condition except for annealing at $58^{\circ} \mathrm{C}$ for $30 \mathrm{~s}$ and extension at $72{ }^{\circ} \mathrm{C}$ for $45 \mathrm{~s}$ was used. Each sample was conducted in three parallels. Standard curves were generated by ten-fold serially diluted plasmids containing the corresponding gene fragments as the template. Negative controls were conducted in which template DNA was replaced by RNase-free water. Melt curve and $1.5 \%$ agarose gel electrophoresis were used to confirm the specificity of the PCR products.

\subsection{High-throughput sequencing and phylogenetic analysis}

PCR amplicons of CAOB amoA were conducted with Illumina Miseq high-throughput Sequencing. The raw data were filtered and the remained sequences were clustered into operational taxonomic unit (OTU) at 97\% nucleotide similarity with QIIME v1.9.1. The representative sequence of each OTU were aligned with the reference sequences collected from NCBI database to construct the Neighbor-Joining tree using 1,000 bootstrap replicates. Diversity indexes and rarefaction curves were also calculated at the OTU level. A principal coordinate analysis (PCoA) of weighted UniFrac distances between samples were calculated to address the community dissimilarities among the different environmental ecosystems. The raw high-throughput Sequencing data have been deposited at NCBI Short Read Archive (SRA) under BioProject PRJNA509063 and BioSample accession numbers SAMN10536240-10536256.

\subsection{Statistical analysis}

Analysis of variance (ANOVA) with Tukey test were carried out to compare different gene abundances and $\mathrm{P}<0.05$ was considered statistically significant. 


\section{Results}

\subsection{The specificity of the primer comamoA $F / R$}

After in-silico and experimental confirmation, the forward primer comamoA $F$ (5'-AGGNGAYTGGGAYTTCTGG-3') and reverse primer comamoA R (5'-CGGACAWABRTGAABCCCAT-3') with the highest specificity and coverage were selected. The primer did not nonspecifically hit any of the 30963 amoA sequences and 31044 pmoA sequences at mismatch of 0 and 1 bases. Even at the mismatch level of 2 bases, the primer could only target $18 \mathrm{amoA}$ and 4 pmoA sequences, demonstrating the great potential to precisely identify the new microbe in complex environments.

\subsection{Abundances of CAOB amoA gene in the five ecosystems}

The abundances of CAOB $a m o A$ gene in the five types of environmental samples were determined via the newly designed primer and the canonical bacterial and archaeal amoA genes were also quantified for comparisons (Fig. 1). Unexpectedly, ubiquitous and abundant CAOB amoA gene were found to be distributed in activated sludge, intertidal zone, river sediment, agricultural soil, and drinking water systems.

The abundance of CAOB amoA gene in activated sludge samples ranged from $5.5 \times 10^{3}$ to $3.2 \times 10^{4}$ copies $\mathrm{ng}^{-1}$ DNA, significantly higher than the canonical AOB amoA genes varying from $9.2 \times 10^{1}$ to $5.6 \times 10^{3}$ copies $\mathrm{ng}^{-1}$ DNA (P < 0.01, Fig. S1). Especially in sample XJH and BB, the ratio of $\mathrm{CAOB}$ to $\mathrm{AOB}$ amoA genes reached to 303.65 and 39.79, respectively. Comparing with the two bacterial amoA genes, the archaeal amoA gene always showed low abundances with an exception of sample BB. The numerical dominances of CAOB over AOB amoA also appeared in the two drinking water samples with $2.5 \times 10^{3}$ and $8.3 \times 10^{1}$ copies $^{-1}{ }^{-1}$ DNA in JN and $\mathrm{BJ}$, respectively. In contrast, opposite results were obtained in soil samples that AOA dominated among the three AOMs with $1.7 \times 10^{4}$ to $4.8 \times 10^{4}$ copies $\mathrm{ng}^{-1} \mathrm{DNA}$, while CAOB amoA gene were significantly lower with ratios of $0.03 \sim 0.60$ to AOA amoA genes $(\mathrm{P}<0.01)$. In the intertidal zone samples, the abundance of CAOB amoA gene in SZ was below detection limit and in SH and TJ they were as low as $3.2 \times 10^{2}$ and $1.6 \times 10^{2}$ copies $\mathrm{ng}^{-1}$ DNA, respectively. In river sediment, the abundances of $\mathrm{CAOB}$ amoA genes also ranged at relatively lower level and showed no significant differences with the canonical bacterial and archaeal amoA genes.

\subsection{Diversity and phylogeny of CAOB amoA gene}

To explore the community structure, high-throughput sequencing was conducted through the newly developed universal PCR primer set targeting both clade A and clade B. A total of 
161,723 sequences were retrieved from the 17 environmental samples and 78 OTUs were clustered excluding the intertidal zone sample SZ1 which was failed in the amplification. The rarefaction curve and Good's coverage (>0.999) indicated the sequencing was nearly to saturation in all the environmental samples (Fig. S2). A neighbor-joining tree comprising 36 OTU representative sequences with proportion higher than $0.5 \%$ was constructed (Fig. 2).

The obtained sequences formed a distinct cluster from the canonical bacterial and archaeal $a m o A$, indicated the specificity of amplification by the current primer comamoA F/R. Consistent with previous results, the sequences were branched into two clades, where 64 out of the 78 OTUs were affiliated with clade A and the other 14 OTUs fell into clade B. The clustering analysis with heatmap visualization revealed the OTU distribution varied significantly between different types of environmental samples (Fig. 3). Moreover, four monophyletic sub-clusters were proposed to be further classified within clade A, among which three clusters were named as $N$. nitrificans, $N$. inopinata, $N$. nitrosa after the first cultured strain names and another one were tentatively named as clade A "soil" cluster given that majority of sequences in this cluster were retrieved from soil environmental samples. Moreover, the phylogenetic tree clearly showed that only clade A was encompassed in activated sludge, intertidal zone and drinking water samples and clade B was only identified in soil and sediment samples (Fig. 4).

At the sub-cluster level, 14 OTUs retrieved from activated sludge were affiliated with cluster $N$. nitrosa which accounted for more than $98.8 \%$ of the total sequences, while the other three clusters only took small proportions in all the activated sludge samples, especially cluster $N$. inopinata was only detected in sample XT with a proportion of $1.8 \%$. In drinking water samples, $93.6 \%$ of the sequences retrieved from sample JN were assigned into cluster $N$. nitrosa while another sample BJ was dominated by the soil cluster $(73.3 \%)$ and $N$. nitrificans cluster (26.7\%). The divergent result was also obtained in the two intertidal zone samples that $N$. nitrosa cluster took up to $98.7 \%$ of the total sequences in sample TJ whereas N. Nitrificans cluster $(73.1 \%)$ and $N$. nitrosa cluster $(19.75 \%)$ dominated in sample SH. The cluster $N$. inopinata was not existed in drinking water and intertidal zone samples.

In sharp contrast to activated sludge, all the soil samples harbored clade B sequences with proportion ranging from $5.7 \%$ to $73.6 \%$ and clade A was dominated by the soil cluster instead of cluster $N$. nitrosa and $N$. nitrificans. PCoA analysis revealed the significant clustering of the four soil samples from other environmental samples (Fig. 5). In the river sediment samples, LZ and SMX showed similar diversity in which clade B accounted for $42.2 \%$ and $16.9 \%$ of their respective sequences, whereas $N$. nitrifican cluster dominated within clade A. However, 
another sample SZ was distinct from LZ and SMX but it showed a much similar structure to activated sludge instead, which was probably attributed to the high ammonia concentration in the severely polluted river in which sample SZ was collected, demonstrating the importance of environmental factors in shaping the microbial community structure.

\section{Discussion}

\subsection{Primer pairs targeting CAOB amoA}

The discovery of comammox radically upended the century-old concept of the two-step nitrification process where ammonia-oxidizing bacteria and archaea mediated ammonia conversion to nitrite. Therefore, the identification and quantification of $\mathrm{CAOB}$ is of great significance to comprehensive and profound understanding of the nitrogen cycle. As CAOB do not form a monophyletic group within Nitrospira lineage II, they cannot be distinguished from the strict nitrite-oxidizing Nitrospira with 16S rRNA based analysis (Pjevac et al., 2017; Koch et al., 2018). Alternatively, the functional amoA gene formed a distinct group from the canonical AOB amoA, which become the best candidate of the specific molecular marker to identify CAOB in complex ecosystems.

Several primer sets have been developed for quantifying CAOB amoA gene using quantitative real-time PCR analyses since their discovery. The divergence of CAOB amoA hindered the development of the universal primer set covering both clades. The primer set targeting only clade A had been designed to quantify CAOB in wastewater treatment systems and other ecosystems (Wang et al., 2018; Xia et al., 2018). The sister primer sets target clade A and clade B were separately developed for identifying CAOB, although nonspecific amplifications were reported (Pjevacet al. 2017; Keene-Beach and Noguera, 2018). Therefore, it was urgent to develop a universal primer pair targeting both clade A and clade B with a higher degeneration to expand the application in various environmental habitats. Herein, the present new universal primer set comamoA F/R possessing high specificity and coverage of CAOB amoA was developed and confirmed by in silico analysis and high-throughput sequencing, which provided an effective and efficient tool for detecting and quantifying $\mathrm{CAOB}$ in complex ecosystems in the follow-up comammox related research.

It was noteworthy that the primer set A189/A682 previously designed for detecting pmoA of methane-oxidizing bacteria (MOB) could amplify a proportion of the new recognized amoA gene due to their close phylogenetic relationship (Dörr et al., 2010; Ho et al. 2016; Holmes et al., 1995). The nonspecific amplification resulted in that hundreds of sequences affiliated with CAOB amoA had been deposited in public NCBI database and incorrectly classified as 
242 canonical pmoA or amoA sequences before the discovery of comammox. Likewise, a number of

243 CAOB amoA sequences were also included in the amoA and $p m o A$ database from FunGene 244 database, suggesting the necessity of filtering the CAOB amoA sequences to ensure the 245 accuracy of the database. In addition, the primer set A189/A682 to identify MOB should be 246 used with caution to avoid the nonspecific amplification of $\mathrm{CAOB} a m o A$ in the future 247 researches.

\section{$248 \quad 4.2$ The ubiquity and diversity of CAOB amoA in various ecosystems}

Ammonia oxidation was recognized as the central and rate-limiting step of nitrogen cycle in environmental ecosystems, which was considered to be driven by the two counterparts AOB and AOA before the discovery of CAOB. Their abundances and activity had been extensively studied to explore their respective role in ammonia oxidation in various ecosystems (Zheng et al., 2017). However, the discovery of CAOB raised new questions regarding their abundances and functions in driving ammonia oxidation, especially comparing with the canonical ammonia oxidizers. Intriguingly, the present results revealed that CAOB amoA genes had significantly higher or comparable abundances with $\mathrm{AOB}$ and $\mathrm{AOA}$ amoA genes in most environmental samples, indicating they were probably an overlooked but significant ammonia oxidizer actively participating in ammonia oxidation.

In WWTPs, AOB was always proposed to be the main contributor to ammonia oxidation due to their dominant numerical abundances over AOA. In the present study similar results were also obtained in most samples with an exception of sample BB that encompassed more abundant archaeal amoA. The preponderance of AOA could be ascribed to the niche specialization of AOA preferring low ammonia concentration, low $\mathrm{pH}$ value and antibiotic or petroleum-containing environment (Zhang et al., 2015; Mussmann et al., 2011; Pan et al., 2018). Unexpectedly, CAOB amoA genes presented higher abundances than $\mathrm{AOB}$ and $\mathrm{AOA}$ amoA genes in all the activated sludge samples, especially in sample $\mathrm{XJH}$ and $\mathrm{BB}$ the ratio of $\mathrm{CAOB}$ to AOB amoA genes reached up to 303.65 and 39.79 , respectively. The prevalence of CAOB in activated sludge was also revealed through the metagenomic survey that $\mathrm{CAOB}$-assigned coding regions accounted for $0.28 \sim 0.64 \%$ of total coding DNA sequences in 16 full-scale WWTPs (Annavajhala et al.., 2018). The large proportion of CAOB in WWTP VetMed was supposed to be the primary contribution to the higher abundance of genus Nitrospira than canonical AOB (Daim et al., 2015). The considerable amount of CAOB among AOMs highlighted that they might take the primary responsibility for ammonia oxidation together with 
275

276

277

278

279

280

281

282

283

activated sludge flocs might be one important factor providing the suitable habitat favoring the CAOB propagation.

Drinking water systems was an ammonia-depleted ecosystem, the existence of ammonia in drinking water can be imported from the source water or artificial addition of chloramine disinfectants to produce biologically stable water for distribution (Regan et al., 2003; van der Wielen et al., 2009). The limited ammonia concentration in drinking water provided suitable substrate concentration for the attached biofilm growth although nitrification in the water distribution systems was unwanted to avoid the chloramine decay (Lawson and Lücker, 2018). An metagenomic analysis revealed the preponderance of CAOB than the canonical AOB and AOA in drinking water samples (Wang et al., 2017b). The high abundance of CAOB was also detected in rapid sand filtration (RSF) system for groundwater treatment (Palomo et al., 2016). It was tempting to speculate that the frequently found numerical dominance of Nitrospira over $\mathrm{AOB}$ and $\mathrm{AOA}$ in drinking water distribution systems was probably ascribed from the abundant $\mathrm{CAOB}$ benefiting from the attached growth on the sand surface provided by the special configuration of RSF system (Gülay et al., 2016; Tatari et al., 2017).

The intertidal zone ecosystem was the ocean-land interaction zone between the low-tide and high-tide line suffering the intense alternative exposure of air and seawater. The abundances of $\mathrm{CAOB}$ amoA in the intertidal samples were relatively low comparing with other ecosystems and became undetectable in the sample SZ. The intense alternative exposure to air would greatly suppress the propagation of $\mathrm{CAOB}$ ascribed to their niche preference of low-oxygen condition. Besides, ocean was proved to be not a suitable habitat as they were absent in oceanic samples, which was probably ascribed from the high salinity (Kuypers, 2017; Santos et al., 2017).

Nitrification process in agricultural soils would result in loss of nitrogen fertilizer and pollution of surrounding waters. The numerical dominances of AOA over AOB were found in majority of researches on soil ecosystems attributed to their higher ammonia affinity and adaption to acidic environments (Kits et al., 2017; Leininger et al., 2006; Nicol et al., 2008). Similar results were also obtained in the present study. CAOB were considered to possess similar ecological niche of low substrate concentration as they both had high affinity to ammonia. However, researches regarding the abundances and functions of $\mathrm{CAOB}$ in soil were still unclear. It was found that CAOB amoA sequences accounted for more than $20 \%$ of all the CuMMO encoded gene using a highly degenerate primer (Wang et al., 2017a). The high abundance of both CAOB amoA clade A and clade B were determined in 300 forest soil samples using the sister primer sets, suggesting they might functionally outcompete the 
canonical AOA under the oligotrophic soil ecosystems (Hu and He, 2017). However, AOA rather than $\mathrm{CAOB}$ were proved to conduct efficient autotrophic nitrification in the forest soil with ${ }^{13} \mathrm{C}$ labelled $\mathrm{CO}_{2}$ microcosm experiment (Shi et al., 2018).

Notably, sequences affiliated with clade B were identified in all the soil samples with highest proportion up to $73.6 \%$, in sharp contrast to activated sludge samples containing not any clade B assigned sequences. Instead, activated sludge samples were highly dominated by $N$. nitrosa cluster within clade A, which was consistent with a previous study on 19 WWTPs using a species-level targeted primer set (Keene-Beach and Noguera, 2018). Besides, the dominance of $N$. nitrosa cluster was also identified in the sediment of a heavily polluted river. Therefore, it seemed that $N$. nitrosa possess the ecological niche of high substrate concentration while clade B preferred to live in ammonia-depleted environments. The divergence might be attributed to the distinct membrane ammonium transporter families harbored by the two CAOB clades that clade A possess Rh-type transporters with low ammonia affinity and high uptake capacity while clade B possess Amt-type transporters with a relatively high ammonia affinity (Palomo et al. 2018). Moreover, the Rh-type ammonia transporters showed high homology (>70\% amino acid similarity) to Nitrosomonas europea (van Kessel, et al., 2015). It was tempting to speculate that $N$. nitrosa cluster had the similar ecological niche with the canonical AOB species adapting to ammonia-rich environments whereas clade B preferred to oligotrophic environments.

The niche specialization and environmental parameters were the dominant factors in shaping the microbial community in various ecosystems (Bahram et al., 2018). However, it is still unclear to which extend the environmental factors such as the process configuration, nutrient concentration and biogeography influence the abundance and diversity of CAOB. Given the potential ecological significance of comammox in the nitrogen cycle, CAOB should be taken into account as one significant AOM in future studies to re-assess the niche specialization and relative contribution together with other nitrifying microorganism in various environments, which is crucial to get a panoramic view of the biogeochemical nitrogen cycle.

\section{Conclusion}

In this study, a primer set targeting both clade A and clade B of CAOB amoA gene was successfully designed. CAOB was found to be widely distributed with significantly higher or comparable abundances compared with canonical AOB and AOA in the investigated environmental samples. The monophyletic clade A and clade B were clustered in the CAOB amoA gene and four sub-clusters were further classified within clade A. The cluster N. nitrosa 
342 dominated CAOB amoA in activated sludge samples while clade B and the new recognized

343 soil cluster within clade A were the primary constitutes in agricultural soils. The distinct

344 communities were supposed to be shaped by niche specialization of different CAOB species

345 and the environmental conditions in various ecosystems. This study provided an effective

346 molecular tool for the following researches on comammox and offered new insights into the

347 ubiquities and community compositions of $\mathrm{CAOB}$ in various environmental ecosystems.

348

\section{Acknowledgements}

350 This work was supported by National Natural Science Foundation [Grant No. 41701278] and

351 Beijing Natural Science Foundation [Grant No. 8174075]. 
352

353

354

355

356

357

358

359

360

361

362

363

364

365

366

367

368

369

370

371

372

373

374

375

376

377

378

379

380

381

382

383

\section{References}

Annavajhala M.K., Kapoor V., Santo-Domingo J., Chandran K. 2018. Comammox Functionality Identified in Diverse Engineered Biological Wastewater Treatment Systems. Environ. Sci. Technol. 5 (2): 110-116.

Bahram M., Hildebrand F., Forslund S.K., Anderson J.L., Soudzilovskaia N.A., Bodegom P.M., Bengtsson-Palme J., Anslan S., Coelho L.P., Harend H., Huerta-Cepas J., Medema M.H., Maltz M.R., Mundra S., Olsson P.A., Pent M., Põlme S., Sunagawa S., Ryberg M., Tedersoo L., Bork P. 2018. Structure and function of the global topsoil microbiome. Nature. 560(7717): 233-237.

Belova S.É., Oshkin IIu., Glagolev M.V., Lapshina E.D., Maksiutov ShSh., Dedysh S.N. 2013. Methanotrophic bacteria in cold seeps of the floodplains of northern rivers. Mikrobiologiia. 82(6): 732-740.

Camejo P.Y., Santo Domingo J., McMahon K.D., Noguera D.R. 2017. Genome-Enabled Insights into the Ecophysiology of the Comammox Bacterium "Candidatus Nitrospira nitrosa". mSystems. 2(5). pii: e00059-17.

Costa E., Pérez J., Kreft J.U. 2006. Why is metabolic labour divided in nitrification? Trends. Microbiol. 14(5): 213-219.

Daims H., Lebedeva E.V., Pjevac P., Han P., Herbold C., Albertsen M., Jehmlich N., Palatinszky M., Vierheilig J., Bulaev A., Kirkegaard R.H., von Bergen M., Rattei T., Bendinger B., Nielsen P.H., Wagner M. 2015. Complete nitrification by Nitrospira bacteria. Nature. 528(7583): 504-509.

Daims H., Lücker S., Wagner M. 2016. A New Perspective on Microbes Formerly Known as Nitrite-Oxidizing Bacteria. Trends. Microbiol. 24(9): 699-712.

Dörr N., Glaser B., Kolb S. 2010. Methanotrophic communities in Brazilian ferralsols from naturally forested, afforested, and agricultural sites. Appl. Environ. Microbiol. 76(4): 1307-1310.

Gülay A., Musovic S., Albrechtsen H.J., Al-Soud W.A., Sørensen S.J., Smets B.F. 2016. Ecological patterns, diversity and core taxa of microbial communities in groundwater-fed rapid gravity filters. ISME J. 10(9): 2209-2222.

Ho A., Lüke C., Reim A., Frenzel. 2016. Resilience of (seed bank) aerobic methanotrophs and methanotrophic activity to desiccation and heat stress. Soil Biol. Biochem. 101: 130-138. 
Holmes A.J., Costello A., Lidstrom M.E., Murrell J.C. 1995. Evidence that particulate methane monooxygenase and ammonia monooxygenase may be evolutionarily related. FEMS Microbiol. Lett .132: 203-208.

Hu H.W., He J.Z. 2017. Comammox — a newly discovered nitrification process in the terrestrial nitrogen cycle. J. Soil. Sediment. 17: 1-9.

Keene-Beach N., Noguera D.R. 2018. Design and assessment of species-level qPCR primers targeting comammox. doi: https://doi.org/10.1101/348664.

Kits K.D., Sedlacek C.J., Lebedeva E.V., Han P., Bulaev A., Pjevac P., Daebeler A., Romano S., Albertsen M., Stein L.Y., Daims H., Wagner M. 2017. Kinetic analysis of a complete nitrifier reveals an oligotrophic lifestyle. Nature. 549(7671): 269-272.

Koch H., Lücker S., Albertsen M., Kitzinger K., Herbold C., Spieck E., Nielsen P.H., Wagner M., Daims H. 2015. Expanded metabolic versatility of ubiquitous nitrite-oxidizing bacteria from the genus Nitrospira. Proc. Natl. Acad. Sci. U S A. 112(36): 11371-11376.

Koch H., van Kessel M.A.H.J., Lücker S. 2018. Complete nitrification: insights into the ecophysiology of comammox Nitrospira. Appl. Microbiol. Biotechnol. doi: 10.1007/s00253-018-9486-3.

Kuypers M.M.M. 2017. Microbiology: A fight for scraps of ammonia. Nature. 549(7671): 162-163.

Lawson C.E., Lücker S. 2018. Complete ammonia oxidation: an important control on nitrification in engineered ecosystems? Curr. Opin. Biotechnol. 50: 158-165.

Leininger S., Urich T., Schloter M., Schwark L., Qi J., Nicol G.W., Prosser J.I., Schuster S.C., Schleper C. 2006. Archaea predominate among ammonia-oxidizing prokaryotes in soils. Nature. 442(7104): 806-809.

Mussmann M., Brito I., Pitcher A., Sinninghe Damsté J.S., Hatzenpichler R., Richter A., Nielsen J.L., Nielsen P.H., Müller A., Daims H., Wagner M., Head I.M. 2011. Thaumarchaeotes abundant in refinery nitrifying sludges express amoA but are not obligate autotrophic ammonia oxidizers. Proc. Natl. Acad. Sci. U S A. 108(40): 16771-16776.

Nicol G.W., Leininger S., Schleper C., Prosser J.I. 2008. The influence of soil pH on the diversity, abundance and transcriptional activity of ammonia oxidizing archaea and bacteria. Environ. Microbiol. 10(11): 2966-2978. 
Palomo A., Jane Fowler S., Gülay A., Rasmussen S., Sicheritz-Ponten T., Smets B.F. 2016. Metagenomic analysis of rapid gravity sand filter microbial communities suggests novel physiology of Nitrospira spp. ISME J. 10(11): 2569-2581.

Pan K.L., Gao J.F., Li H.Y., Fan X.Y., Li D.C., Jiang H. 2018. Ammonia-oxidizing bacteria dominate ammonia oxidation in a full-scale wastewater treatment plant revealed by DNA-based stable isotope probing. Bioresour. Technol. 256: 152-159.

Pester M., Rattei, T., Flechl, S., Gröngröft, A., Richter. A., Overmann, J., Reinhold-Hurek, B., Loy, A., Wagner, M. 2012. amoA-based consensus phylogeny of ammonia-oxidizing archaea and deep sequencing of amoA genes from soils of four different geographic regions. Environ. Microbiol. 14: 525-539.

Pjevac P., Schauberger C., Poghosyan L., Herbold C.W., van Kessel M.A.H.J., Daebeler A., Steinberger M., Jetten M.S.M., Lücker S., Wagner M., Daims H. 2017. AmoA-Targeted Polymerase Chain Reaction Primers for the Specific Detection and Quantification of Comammox Nitrospira in the Environment. Front. Microbiol. 8: 1508.

Regan J.M., Harrington G.W., Baribeau H., Leon R.D., Noguera D.R. 2003. Diversity of nitrifying bacteria in full-scale chloraminated distribution systems. Water Res. 37(1): 197-205.

Rotthauwe J.H., Witzel K.P., Liesack W. 1997. The ammonia monooxygenase structural gene amoA as a functional marker: molecular fine-scale analysis of natural ammonia-oxidizing populations. Appl. Environ. Microbiol. 63: 4704-4712.

Santos J.P., Mendes D., Monteiro M., Ribeiro H., Baptista M.S., Borges M.T., Magalhaes C. 2017. Salinity impact on ammonia oxidizers activity and amoA expression in estuarine sediments. Estuar. Coast. Shelf S. 211: 177-187.

Shi X., Hu H., Wang J., He J., Zheng C., Wan X., Huang Z. 2018. Niche separation of comammox Nitrospira and canonical ammonia oxidizers in an acidic subtropical forest soil under long-term nitrogen deposition. Soil Biol. Biochem. 126: 114-122.

Tatari K., Musovic S., Gülay A., Dechesne A., Albrechtsen H.J., Smets B.F. 2017. Density and distribution of nitrifying guilds in rapid sand filters for drinking water production: Dominance of Nitrospira spp. Water Res. 127: 239-248. 
van der Wielen P.W., Voost S., van der Kooij D. 2009. Ammonia-oxidizing bacteria and archaea in groundwater treatment and drinking water distribution systems. Appl. Environ. Microbiol. 75(14): 4687-4695.

van Kessel M.A., Speth D.R., Albertsen M., Nielsen P.H., Op den Camp H.J., Kartal B., Jetten M.S., Lücker S. 2015. Complete nitrification by a single microorganism. Nature. 528(7583): 555-559.

Wang J.G., Xia F., Zeleke J., Zou B., Rhee S.K., Quan Z.X. 2017a. An improved protocol with a highly degenerate primer targeting copper-containing membrane-bound monooxygenase genes for community analysis of methaneand ammonia-oxidizing bacteria. FEMS Microbiol. Ecol. 93(3). pii: fiw244.

Wang M., Huang G., Zhao Z., Dang C., Liu W., Zheng M. 2018. Newly designed primer pair revealed dominant and diverse comammox amoA gene in full-scale wastewater treatment plants. Bioresour. Technol. 270:580-587.

Wang Y., Ma L., Mao Y., Jiang X., Xia Y., Yu K., Li B., Zhang T. 2017b. Comammox in drinking water systems. Water Res. 116: 332-341.Xia F., Wang J.G., Zhu T., Zou B., Rhee S.K., Quan Z.X. 2018. Ubiquity and diversity of complete ammonia oxidizers (comammox). Appl. Environ. Microbiol. pii: AEM.01390-18.

Xia F., Wang J.G., Zhu T., Zou B., Rhee S.K., Quan Z.X. 2018. Ubiquity and diversity of complete ammonia oxidizers (comammox). Appl. Environ. Microb. 84: e01390-18.

Zhang Y., Tian Z., Liu M., Shi Z.J., Hale L., Zhou J., Yang M. 2015. High concentrations of the antibiotic spiramycin in wastewater lead to high abundance of ammonia-oxidizing archaea in nitrifying populations. Environ. Sci. Technol. 49(15): 9124-9132.

Zheng M., Wang M., Zhao Z., Zhou N., He S., Liu S., Wang J., Wang X. 2018. Transcriptional activity and diversity of comammox bacteria as a previously overlooked ammonia oxidizing prokaryote in full-scale wastewater treatment plants. Sci. Total. Environ. 656: 717-722.

Zheng M.S., Fu H.Z., Ho Y.S. 2017. Research trends and hotspots related to ammonia oxidation based on bibliometric analysis. Environ. Sci. Pollut. R. 24: 20409-20421. 
Table 1 The characteristics of the environmental samples used in this study.

\begin{tabular}{|c|c|c|c|c|c|}
\hline Sample types & $\begin{array}{c}\text { Sample } \\
\text { ID }\end{array}$ & Longitude and latitude & $\begin{array}{c}\text { Sampling } \\
\text { Date }\end{array}$ & $\mathrm{pH}$ & $\mathrm{NH}_{4}-\mathrm{N}^{\mathrm{a}}$ \\
\hline \multirow[t]{6}{*}{$\begin{array}{l}\text { Activated } \\
\text { sludge }\end{array}$} & $\mathrm{XJH}$ & $40^{\circ} 01^{\prime 2} 25^{\prime \prime}, 116^{\circ} 18^{\prime} 20^{\prime \prime}$ & Sep-16 & 7.25 & 29.8 \\
\hline & YQ & $40^{\circ} 27^{\prime} 18^{\prime \prime}, 115^{\circ} 58^{\prime} 24^{\prime \prime}$ & Sep-16 & 7.76 & 21.3 \\
\hline & YC & $34^{\circ} 25^{\prime} 34^{\prime \prime}, 112^{\circ} 26^{\prime} 10^{\prime \prime}$ & Oct-16 & 7.2 & 24.4 \\
\hline & YS & $34^{\circ} 43^{\prime} 10^{\prime \prime}, 112^{\circ} 47^{\prime} 07^{\prime \prime}$ & Oct-16 & 8.0 & 50.0 \\
\hline & $\mathrm{XT}$ & $27^{\circ} 49^{\prime} 57^{\prime \prime}, 112^{\circ} 54^{\prime} 09^{\prime \prime}$ & Oct-16 & 7.39 & 10.4 \\
\hline & BB & $32^{\circ} 55^{\prime} 54^{\prime \prime}, 117^{\circ} 24^{\prime} 23^{\prime \prime}$ & Oct-16 & 7.5 & 27.1 \\
\hline \multirow[t]{4}{*}{$\begin{array}{l}\text { Agricultural } \\
\text { soil }\end{array}$} & SD & $32^{\circ} 59^{\prime} 34^{\prime \prime}, 114^{\circ} 01^{\prime} 46^{\prime \prime}$ & Oct-16 & 5.5 & 18.2 \\
\hline & $\mathrm{JZ}$ & $38^{\circ} 02^{\prime} 37^{\prime \prime}, 115^{\circ} 02^{\prime} 14^{\prime \prime}$ & Oct-16 & 6.5 & 10.0 \\
\hline & GZ & $37^{\circ} 04^{\prime} 36^{\prime \prime}, 115^{\circ} 07^{\prime} 56^{\prime \prime}$ & Oct-16 & 5.5 & 7.7 \\
\hline & SH1 & $31^{\circ} 16^{\prime} 10^{\prime \prime}, 121^{\circ} 26^{\prime} 29^{\prime \prime}$ & Oct-16 & 5 & 15.8 \\
\hline \multirow[t]{3}{*}{ River sediment } & SZ & $22^{\circ} 33^{\prime} 44^{\prime \prime}, 114^{\circ} 03^{\prime} 17^{\prime \prime}$ & Aug-17 & N.A. & N.A. \\
\hline & $\mathrm{LZ}$ & $36^{\circ} 04^{\prime} 47^{\prime \prime}, 103^{\circ} 49^{\prime} 32^{\prime \prime}$ & Oct-16 & 7.54 & 5.4 \\
\hline & SMX & $34^{\circ} 47^{\prime} 50^{\prime \prime}, 111^{\circ} 13^{\prime} 04^{\prime \prime}$ & Oct-16 & 7.38 & 3.8 \\
\hline \multirow[t]{2}{*}{ Drinking water } & $\mathrm{JN}$ & $35^{\circ} 26^{\prime} 05^{\prime \prime}, 116^{\circ} 35^{\prime} 05^{\prime \prime}$ & Oct-17 & N.A. & N.A. \\
\hline & $\mathrm{BJ}$ & $40^{\circ} 07^{\prime} 00^{\prime \prime}, 116^{\circ} 14^{\prime} 01^{\prime \prime}$ & Sep-17 & N.A. & N.A. \\
\hline \multirow[t]{3}{*}{ Intertidal zone } & $\mathrm{SH}$ & $31^{\circ} 20^{\prime} 24^{\prime \prime}, 121^{\circ} 39^{\prime} 27^{\prime \prime}$ & Jul-17 & N.A. & N.A. \\
\hline & $\mathrm{SZ1}$ & $22^{\circ} 30^{\prime} 47^{\prime \prime}, 114^{\circ} 02^{\prime} 42^{\prime \prime}$ & Jul-17 & N.A. & N.A. \\
\hline & $\mathrm{TJ}$ & $39^{\circ} 06^{\prime} 04^{\prime \prime}, 117^{\circ} 44^{\prime} 26^{\prime \prime}$ & Aug-17 & N.A. & N.A. \\
\hline
\end{tabular}



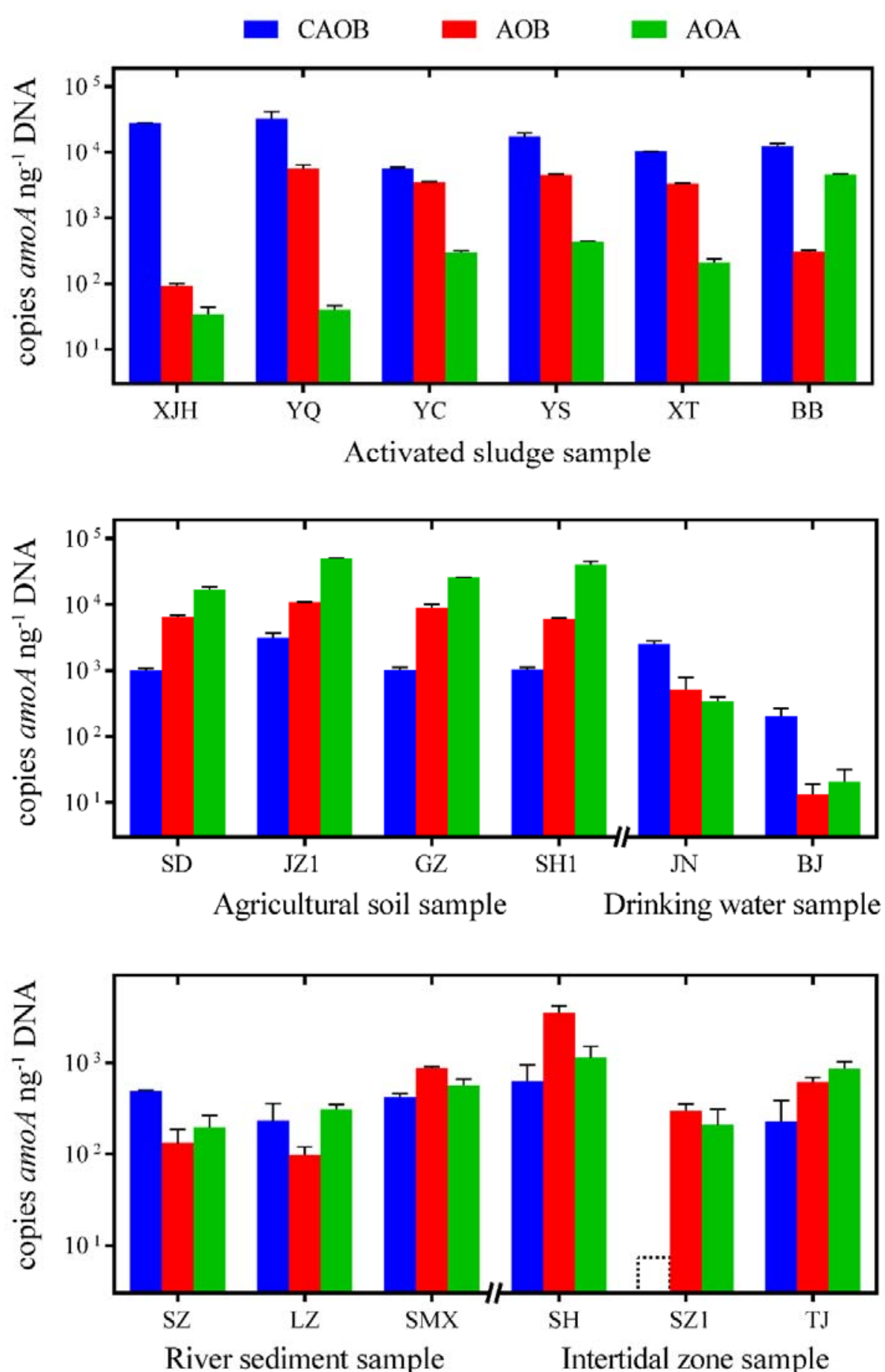

Fig. 1 Abundances of amoA gene from $\mathrm{CAOB}, \mathrm{AOB}$ and $\mathrm{AOA}$ in the 18 environmental samples. The dotted line in sample SZ1 represents below detection limit. 


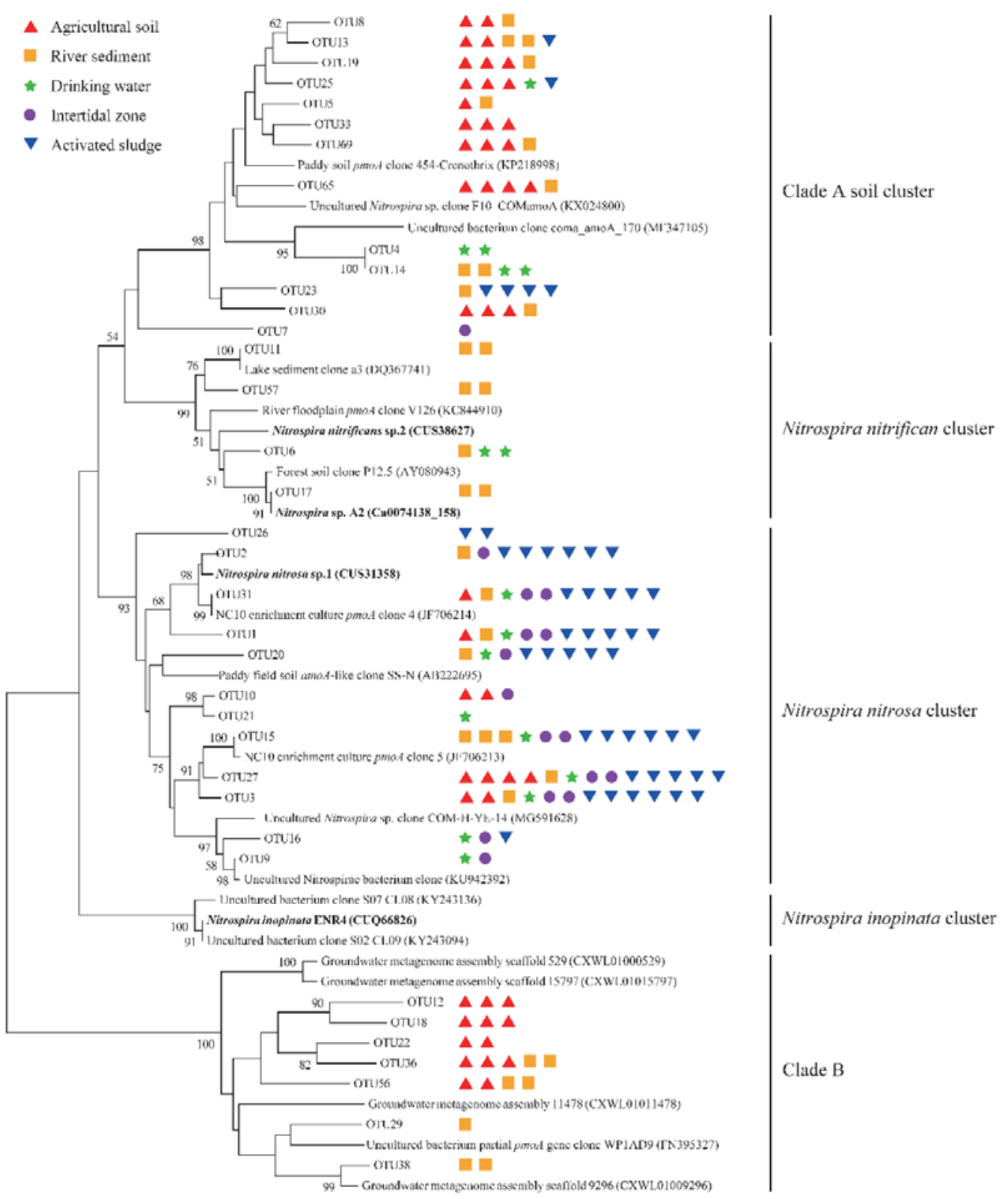

0.05

Fig. 2 Neighbor-joining consensus tree generated from an alignment of CAOB amoA reference sequences and representative sequences of the top 36 OTU with proportion higher than $0.5 \%$ retrieved from the environmental samples. The bootstrap values higher than $50 \%$ are indicated at branch points. The scale bar represents $5 \%$ nucleic acid sequence divergence. 


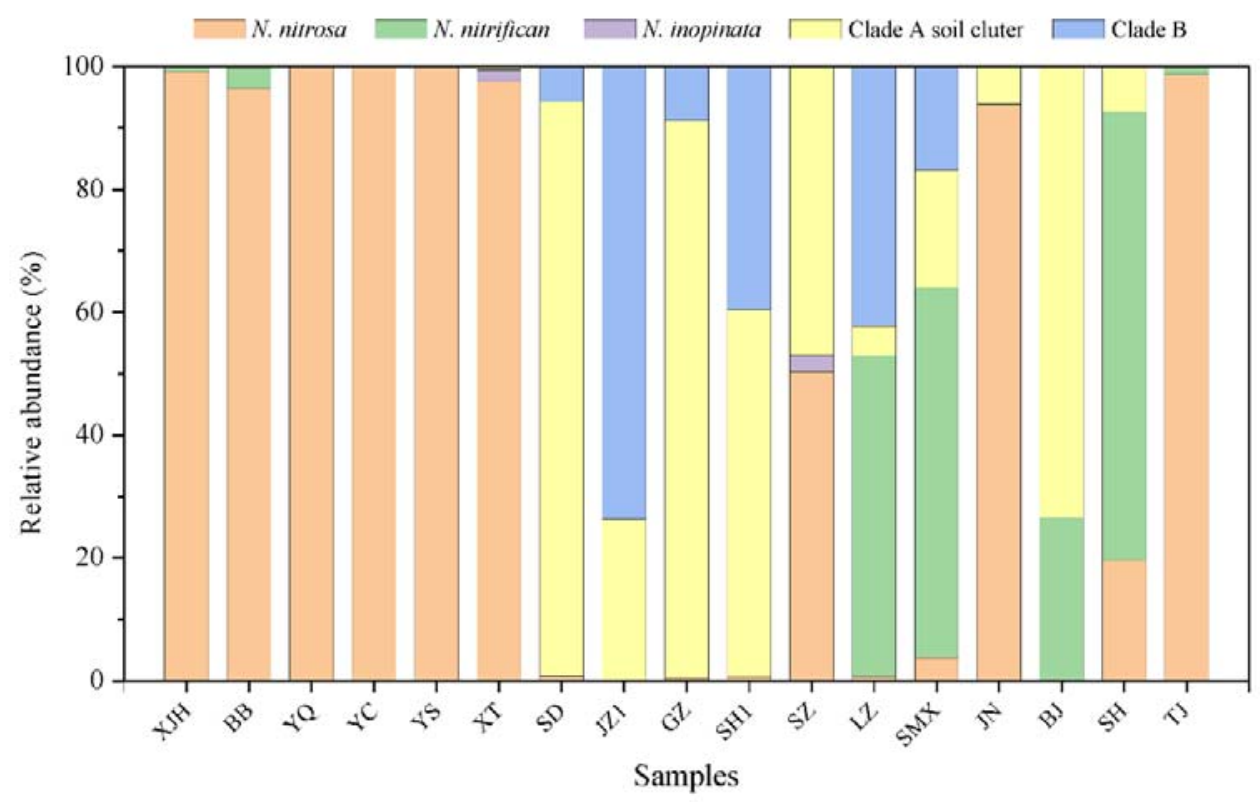

Fig. 3 Distributions and relative abundances of different phylogenetic clusters of CAOB amoA in the 17 environmental samples. The amplification of intertidal zone sample SZ was failed and not shown. 


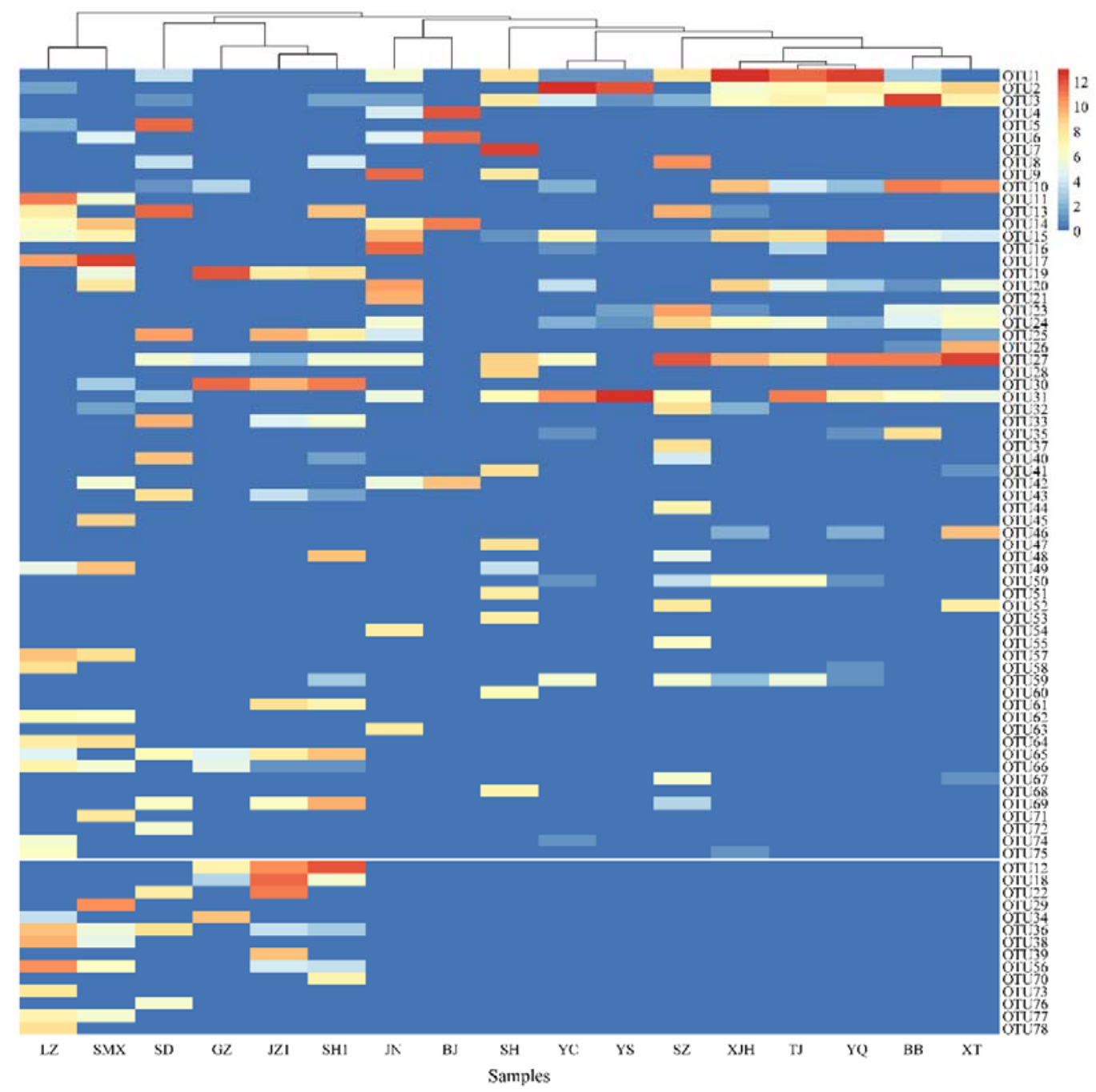

Fig. 4 Heatmap displying the relative abundances of the 78 OTUs retrieved from the environmental samples. OTUs affilated with clade A and clade B were depicted above and below the white line respectively. 


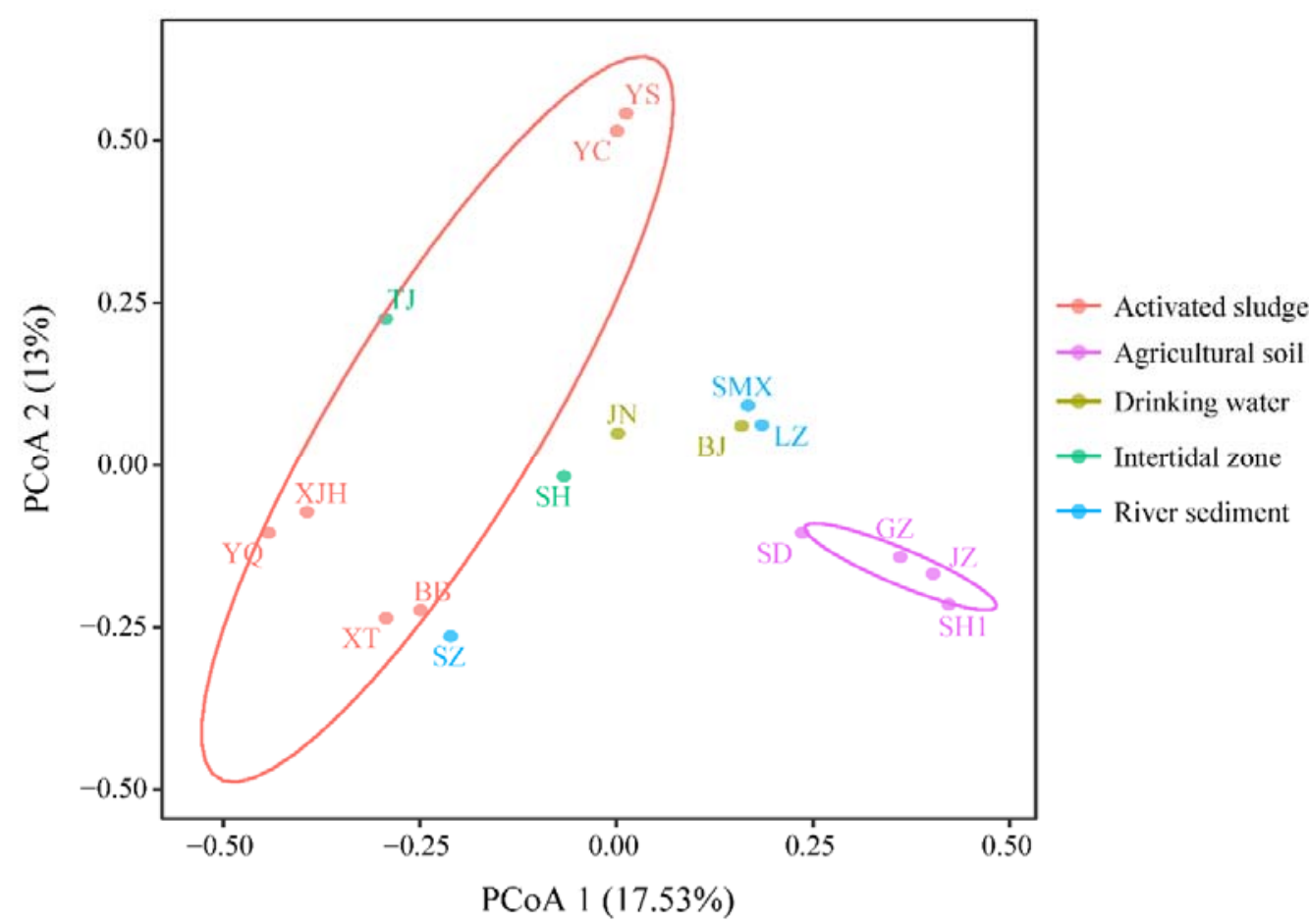

Fig. 5 Principal coordinates analysis (PCoA) ordinations of the CAOB amoA community of the 17 environmental samples. Data points are colored by the different types of environmental ecosystems. 